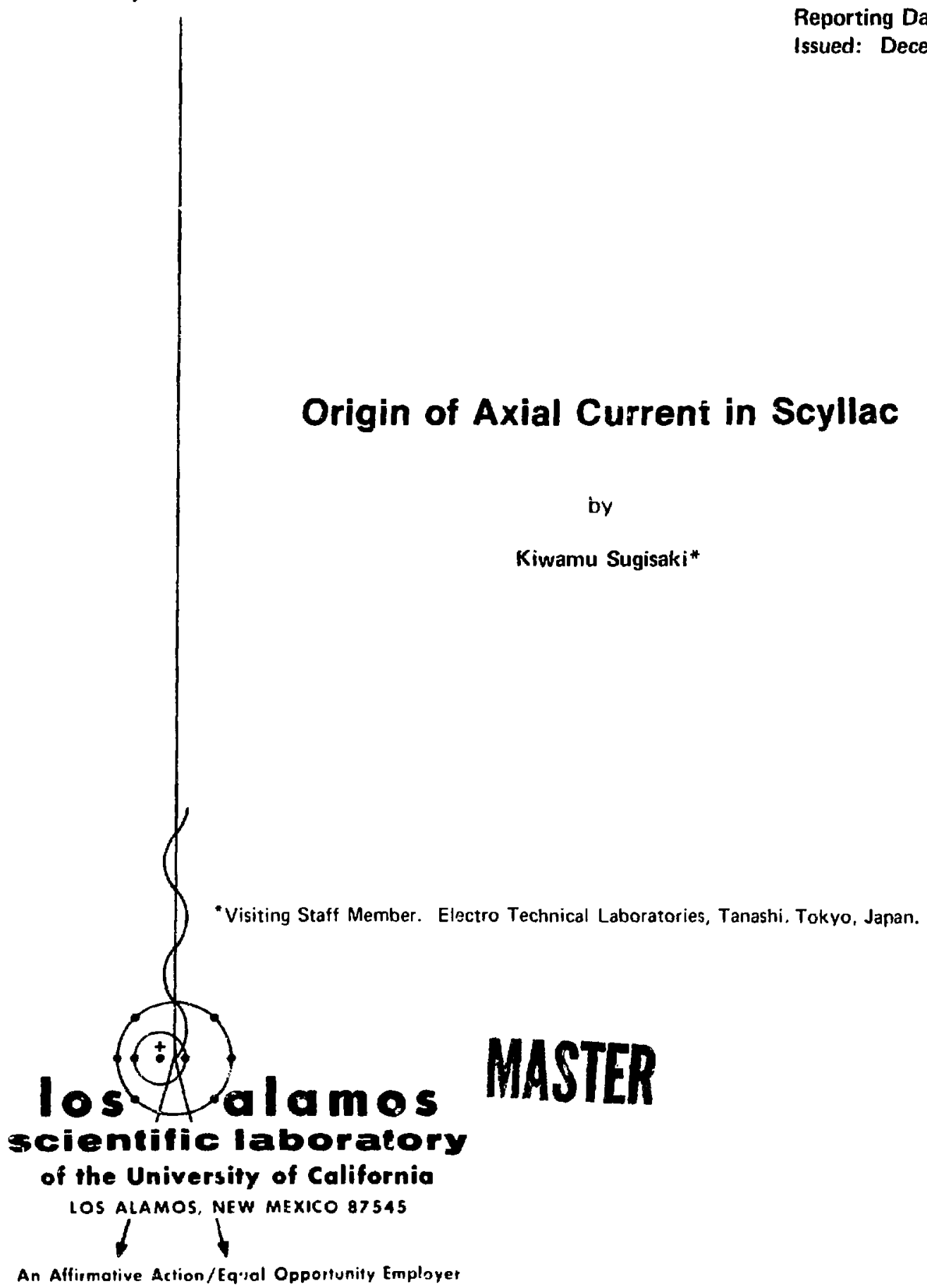

Reporting Date: December 1975

Issued: December 1975

\title{
Origin of Axial Current in Scyllac
}

\author{
Kiwamu Sugisaki*
}

An Alfirmotive Action/Eq'sal Opportunity Employer 
In the interest of prompt distribution, this report was not edited by the Technical Information staff.

Printed in the L'nited Situtes of Amerien. Available Irum Silticunal 'Technical Infirmation Service'

I.S. Deprartment of Commerse

itzesis Port Roval Puad

Siprintield VA 22151

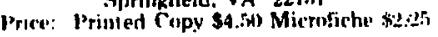

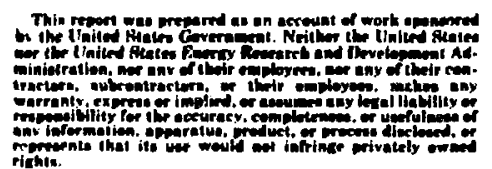

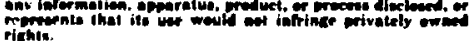


ORIGIN OF AXIAL CURRENT IN SCYLLAC

by

Kiwanu Sugisaki
Thus iepurt was Grepured as an sccount of wutk

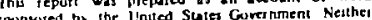
the United Stures nol the Uniled State F.migy Rewarch and Development Adminustration, hior ans of uneis empluyees, mert any of theu contsactors. subcontractots of thes empluyets. makes any watranty, expless of impled, ni asumes any legen

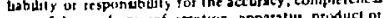
ins uscfulnes of any imfentration, apparatu, product mo intrigt paratel, owned sukhts

\section{ABSTRACT}

The origin of the axial current oboerved in Scyllac (a high beta stellarator experiment) is discussed. A shaped coil and/or helical winding produce rotational transform which links magnetic lines of force to the plasma column and the axial current is induced electromagnetically. This phenomenon is inherent in a pulsed high-beta stellarator. The rotational transform produced by the induced axial current 18 much smaller than that associated with the $\ell=1,0$ equilibrium fields. The ef fect of the axial current on the equilibrium and stability of the plasma column is thus small. It is also shown that the magnetic field shear near a plasma surface is very strong.

\section{INTRODUCTION}

An axial plasma current of $1 \sim 1.5 \mathrm{kA}$ peak amplitude is observed in the Scyllac full torus experiments. ' The amplitude of the current exceeds che Kruskal - Shafranov $11 \mathrm{~m} 1 \mathrm{t}$ at a radius of $1 \mathrm{~cm}$ ( $500 \mathrm{~A})$. Therefore, the kink Instability is posstble. However, the gross behavior of the plasma column appears to be unchanged, Irrespective of whether the net axial current is reduced tu zero by means of an externally induced axial current. This effect is not inconsistent with the small theoret1cal growth rate of the kink instability.

In this report, the origin of the axial current is discussed in terms of rotational transform. Rotational transform is produced with a shaped comp"esston coll and/or helical windings. Magnetic lines of forc? link the toroidal plasma column. If the plasma is produced in a pulsed fashion, linkage of the magnetic lines of force leads t. electromagnetic induction along the axis. The magnitude of the Iinkage can be obtained from the rotational transform, and determines the magnitude of the axial current.

II. AXIAL CURRENT

Rotational transform, is defined as?

$$
1(i)=2 \pi \frac{d X}{d \phi}
$$

where $X$ is the azimuthal magnetic flux and $\Phi$ is the longitudinal flux. We assume that the longitujinal component of the magnetic field is constant in the plasma and in the surrounding vacuum and is much larger than the azimuthal component. Then the longitudinal flux $\Phi$ is, in lowest order,

$$
\Phi=\pi a^{2} B_{i}+\pi\left(r^{2}-a^{2}\right) B_{0}
$$

where $B_{1}$ and $B_{0}$ are the magnetic fleld in the plasma and in the vacuum, respectively, and $a$ is the average radius or the plasma column. The azimuthal flux $x$ outside the plasma column 1 expressed as

$$
Y(r) \approx H_{0} j_{a}^{r} 1(r) r d r
$$

The azimuthal flux $X$ is the magnetic flux per unt length which links the plasma colunn and produces electromagnetic induction along the exis if the magnetic field is applied abruptly. The resultant induced axidl current I has the form

$$
I \approx-\frac{X(b)}{R / h \ln b / a} \cdot \frac{B_{0}}{\mu \ln b / a} \int_{a}^{b} 1(r) r d r
$$


where $2 \pi / h$ is the wavelength of the helical $\ell=1$ magnetic fleld and $b$ is the average wall radius of che shaped compression $\operatorname{co} 11 .{ }^{3}$ The magnitude of the axlal current is proportional to the rotational cransform 1 , and is induced in such a manner as to reduce che original rotational transform produced with the shaped coll.

\section{I. ROTATIONAL TRANSFORM}

The magnetic field in the vacuum region is calculated from

$$
\begin{aligned}
& \vec{B}=\vec{\nabla}_{\phi} \\
& \Delta \phi=0
\end{aligned}
$$

with boundary conditions

$$
\begin{aligned}
& \vec{\nabla}_{f_{1}} \cdot \vec{\nabla}_{\phi}=0 \\
& \vec{\nabla}_{2} \cdot \vec{\nabla}_{\phi}=0
\end{aligned}
$$

The quantities $f_{1}$ and $f_{2}$ describe the Inner surface of the shaped compression coll and the outer surface of the plasma column respectively:

$f_{1}(r, \theta, z)=r-b-\Delta_{l=1} \cos (\theta+h z)-\Delta_{l=0} \cos (h z)=0$

$\tau_{2}(r, \theta, z)=r-a-\delta_{\ell=1} \cos (\theta+h z)-\delta_{\ell=0} \cos (h z)=0$

The last terms of $f_{1}$ and $f_{2}$ do not contribute to the following discussion and are omitted hereafter. Toroldal effects are also 1gnored. For simplicity, we further assume that

$$
\begin{aligned}
& \Delta_{\ell=1} / b \ll 1 \\
& \delta_{\ell=1} / a \ll 1
\end{aligned}
$$

The first assumption in Eq. (8) is satisfied experimentally in Scyllac but the second is not. The solution of Eq. (5) 1s, in lowest order,

$=\mathrm{B}_{\mathrm{o}} \mathrm{T}+\frac{\mathrm{l}}{\mathrm{h}}\left[\mathrm{CI}_{1}(\mathrm{hr} r)+\mathrm{DK}_{1}(\mathrm{hr} r)\right] \sin (\theta+\mathrm{h} z)$

where

$$
\begin{aligned}
c & =h B_{0} \frac{\delta_{l=1} K_{1}^{\prime}(h b)-\Delta_{l=1} K_{1}^{\prime}(h a)}{I_{1}^{j}(h b) K_{1}^{\prime}(h a)-I_{1}^{\prime}(h a) K_{l}^{\prime}(h b)} \\
& \approx-2 h B_{0} \frac{b^{2} \Delta_{\ell=1}-a^{2} \delta_{l=1}}{b^{2}-a^{2}}
\end{aligned}
$$

$$
\begin{aligned}
D & =h B_{0} \frac{\Delta_{\ell=1} I_{1}^{\prime}(h a)-\delta_{\ell-1} I_{1}^{\prime}(h b)}{I_{1}^{\prime}(h b) K_{1}^{\prime}(h a)-I_{1}^{\prime}(h a) K_{1}^{\prime}(h b)} \\
& \approx-h B_{0}\left(\frac{h^{2} a^{2} b^{2}}{b^{2}-a^{2}}\right)\left(\Delta_{\ell=1}-\delta_{\ell=1}\right)
\end{aligned}
$$

for ha, hb $\ll 1$. The rotational transform per $\ell=1$ wavelength is calculated from the method of averaging 4 and 1s, in lowest order,

$$
\begin{aligned}
I(r) & =\frac{\pi}{B_{0}^{2}}\left\{C^{2}\left(\frac{I_{1}^{\prime}(x)}{x}\right)^{2}\left[1-2 I^{1}+\left(1+x^{2}\right)\left(I^{1}\right)^{2}\right]\right. \\
& +2 C D \frac{I_{1}^{\prime}(x)}{x} \frac{K_{1}^{\prime}(x)}{x}\left[1-I^{1}-K^{1}+\left(1+x^{2}\right) I^{1} K^{1}\right] \\
& \left.+D^{2}\left(\frac{K_{1}^{\prime \prime}(x)}{x}\right)^{2}\left[1-2 K^{1}+\left(1+x^{2}\right)\left(K^{1}\right)^{2}\right]\right\}(11)
\end{aligned}
$$

where

$$
\begin{aligned}
& I^{1}=\frac{I_{1}(x)}{x I_{1}^{\prime}(x)} \\
& K^{\prime}=\frac{K_{1}(x)}{x K_{1}^{\prime}(x)} \\
& x=h r
\end{aligned}
$$

For ha, hb 1, Eq. (II) reduces to

$$
f(r) \approx \frac{\pi h^{2} \Delta^{2} l=1}{1-a^{2} / b^{2}}\left\{\left(1-\frac{a^{2}}{b^{2}} \frac{\delta_{\ell=1}}{\Delta_{\ell=1}}\right)^{2}+\left(1-\frac{a^{1}}{b^{2}} \frac{\delta_{l=1}}{\Delta_{l=1}}\right)\right.
$$

$\left.x \frac{\mathrm{a}^{2}}{\mathrm{r}^{2}}+\frac{4}{\mathrm{~h}^{2} \mathrm{a}^{2}}\left(\mathrm{I}-\frac{\delta_{l=1}}{\Delta_{l=1}}\right)^{2} \frac{\mathrm{a}^{6}}{\mathrm{r}^{6}}\right\}$

The first term In Eq. (12) is the rotational transform of the vacuum magnetic field and the other terms are those reculted from the presence of the plasma. When the beta value of the plasma is large and $\Delta_{\ell=1}$ is not equal to $\delta_{\ell=1}$, the last term is dominant and the rotational transform with the plasma is much larger than that without the plasma. Substitution of Eq. (12) Into Eq. (4) gives the magnttude of the induced axial current as 


$$
\begin{aligned}
& I \approx \frac{M B_{o}}{\mu l n b / a} \frac{h^{3} \Delta_{l=1}^{2} b^{2}}{\left(1-\frac{a^{2}}{b^{2}}\right)^{2}}\left\{\frac{2}{2}\left(1-\frac{a^{2}}{b^{2}} \frac{\delta_{\ell=1}}{\Delta_{\ell=1}}\right)^{2}\right. \\
& x\left(1-\frac{a^{2}}{b^{2}}\right)+\frac{a^{2}}{b^{2}}\left(1-\frac{a^{2}}{b^{2}} \frac{\delta_{l=1}}{\Delta_{\ell=1}}\right) \text { e b/a }+\frac{1}{h^{2} b^{2}} \\
& \left.x\left(1-\frac{\delta_{\ell=1}}{\Delta_{\ell=1}}\right)^{2} \quad\left(1-\frac{a^{4}}{b^{4}}\right)\right\} \\
& \approx \frac{m B_{0} \Delta_{\ell=1}^{2}}{\mu \ln b / a}\left(1-\frac{\delta_{\ell=1}}{\Delta_{\ell=1}}\right)^{2}
\end{aligned}
$$

for $\delta_{\ell=1} \neq \Delta_{\ell=1}$. For the parameters $B_{o}=40 \mathrm{kG}$, $h=15 \mathrm{~m}^{-1}, \Delta_{\ell=1}=0.43 \mathrm{~cm}, \delta_{\ell=1}=0 \mathrm{~cm}$, \& b $/ a=2$, we find $I=1.4 \mathrm{kA}$. The value is compatible with the experimental value.' The time evolution and more exact magnitude of I are obtained by taking into consileration that the plasma radius a and distortio's of the plasma column $\delta_{D,=1}$ are time dependent quantities. As we have noted above, the direction of the axial current is oriented in such a manner as to reduce the ortginal rotational transform and dependent on whether the helical $\hat{i}=1$ magnetic fleld rotates clockwise or counterclockwise along the line of force. If the helical $\ell=1$ field rotates clockwise as in Scyllac, the axtal current is induced in antiparalle) with the line of force.

Now we examine to what extent the axial curient affects the original magnetic field. This is cotiventiently carried out in terms of rotational transiorm. The rotational transform $1_{c}(r)$ associated with the axtal current is given by

$$
i_{c}(r) \approx \frac{i I}{h r^{2} B_{o}} \approx \frac{1}{2 \ln b / a} \frac{\Lambda_{l=1}^{2}}{r^{2}}
$$

at radius $r$. The ratio of the original rotational transform 1(a) to the curient produced rotational transform $1_{c}(a)$ on the plasma surface indicates the influence of the axial current:

$$
\frac{1_{c}(a)}{1(a)} \approx \frac{1}{4 \ln b / a} \sim 10 \%
$$

for the present Scyllac parameters. Therefore, we conclude that the induced axial current has little influence on the original magnetic fleld. Equation (15) 1s eas1:? understood from the fact that $11 \mathrm{nk}$ age of the magnetic field to the plasma column is localized near the plasma surface, as seen in Eq. (12). However, the axial current observed in Scyllac exceeds the Kruskal-Shafranov limiting current at the radius of $1 \mathrm{~cm}$. This is explained by the fact that the total rotationai transform in Scyllac 1s much larger than $2 \pi$. From Eq. (12), we obtain

$$
i(a) \approx \frac{4 \pi \Delta^{2} l=1}{a^{2}}\left(1-\frac{\delta_{l=1}}{\Delta_{l=1}}\right)^{2} \approx \frac{\pi \beta^{2} \varepsilon_{\ell=1}^{2}}{a^{2}}
$$

where we have used the equilibrlum condicion

$$
\frac{\delta_{l=1}}{\Delta_{\ell=1}} \approx \frac{1}{1-\beta / 2\left(1-a^{2} / b^{2}\right)} \approx \frac{1}{1-\beta / 2}
$$

The quantsty $\beta$ is the plasma beta in our sharp boundary model. The plasma radius a in the Scyllac experiments 1s comparable to the distortion of the plasma column $\delta_{\ell=1}$. Then the rotational transform $i(a)$ per the wavelength of the helical $\ell=1$ magnetic field is of the order of 1 in the presence of a high beta plasma, while the vacuum rotational transfo:m is or tile order of $\left(h \Delta_{l=1}\right)^{2}$.

We find from $E q$. (12) that the magnetlc field shear near the plasma surface is very strong. The shear parameter $\theta$ is given as

$$
0=\frac{d i}{d r} \frac{h r^{2}}{2 r} \approx-\frac{12 \Delta_{l=1}^{2} a^{4} h}{r^{5}} \sim-1 .
$$

\section{CONCLUDINS REMARKS}

We have shown that tha axial current in Scyllac results from rotational transform. Therefore, any procedure which varies rotational transform, such as dynamic stabilization, lead to the additional current induction. However, the induced axtal current has little effect on the original (not vacuum :) rotaitonal transform.

Rotational transform and magnetic field shear in Scyllac are also calculated. The strong shear calculated near the plasma surface suggests that flute-like instabilities with $m \geq 2$ are suppressed for Scyllac parameters, even in the cases where finfte Larmor radius (FLR) effects are weak, such as in a staged theta pinch. 
In this study, we have Ignored the effect of the magnetic field inside the plasma column. Thto effect does not seem to be Important for a high beta plasma.

G. Miller has obtained a result similar to Eq. (13) by considering the motion of the plasma colum during the aetting-up phase."

ACKNOWLEDGMENT

This work was carried out at the suggegtion of W. R. Ellis. The author is grateful to W. R. Ellis for reading the manuscript in an earlier version and thanks the members of the CTR Division at Los Alamos Scientific Laboratory for their haspitality.

\section{REFERENCES}

1. E. L. Cantrell and others, Proceedings of the Fifth IAEA Conference on Plasma Physlcs and Controlled Nuclear Fusion Research (Tokyo, Japan, November 11-15, 1974, to the published) also Los Alamos Sclentffic Laboratory Report, LA-UR-74-1549 (1974).

2. B. B. Kadontsev, Soviet Phys. JETP 10, 1167 (1959).

3. W. R. Ell1s and others, Nuclear Fusion 14, 841 $(1974)$.

4. A. I. Morozov and L. S. Solov'ev, "Revlew of Plasma Phystes", Vol, 2, Pp, 22-27, Consultants Bureau, New York, 1965.

5. G. Miller, Phys. Fluids, (to be published). 